\title{
APOGEE spectroscopic evidence for chemical anomalies in dwarf galaxies: The case of M 54 and Sagittarius
}

\author{
José G. Fernández-Trincado ${ }^{1,2,3 \star(\mathbb{D}) \text {, Timothy C. Beers }}{ }^{4}$ (D), Dante Minniti ${ }^{5,6}$, Christian Moni Bidin ${ }^{7}$, Beatriz \\ Barbuy $^{8}$, Sandro Villanova ${ }^{9}$, Doug Geisler ${ }^{9}{ }^{10,11}$, Richard R. Lane ${ }^{2}$, Alexandre Roman-Lopes ${ }^{10}$ (D) and Dmitry \\ Bizyaev $^{12,13}$
}

1 Institut Utinam, CNRS UMR 6213, Université Bourgogne-Franche-Comté, OSU THETA Franche-Comté, Observatoire de Besançon, BP 1615, 25010 Besançon Cedex, France

2 Instituto de Astronomía y Ciencias Planetarias, Universidad de Atacama, Copayapu 485, Copiapó, Chile

3 Centro de Investigación en Astronomía, Universidad Bernardo O Higgins, Avenida Viel 1497, Santiago, Chile

${ }^{4}$ Department of Physics and JINA Center for the Evolution of the Elements, University of Notre Dame, Notre Dame, IN 46556, USA

5 Depto. de Cs. Físicas, Facultad de Ciencias Exactas, Universidad Andres Bello, Av. Fernández Concha 700, Las Condes, Santiago, Chile

6 Vatican Observatory, V00120 Vatican City State, Italy

7 Instituto de Astronomía, Universidad Católica del Norte, Av. Angamos 0610, Antofagasta, Chile

8 Universidade de São Paulo, IAG, Rua do Matão 1226, Cidade Universitária, São Paulo 05508-900, Brazil

9 Departamento de Astronomía, Casilla 160-C, Universidad de Concepción, Concepción, Chile

${ }_{10}$ Departamento de Astronomía, Universidad de La Serena, 1700000 La Serena, Chile

11 Instituto de Investigación Multidisciplinario en Ciencia y Tecnología, Universidad de La Serena. Benavente 980, La Serena, Chile

12 Apache Point Observatory and New Mexico State University, Sunspot, NM, 88349, USA

13 Sternberg Astronomical Institute, Moscow State University, Moscow, Russia

Received ...; Accepted ...

\begin{abstract}
We present evidence for globular cluster stellar debris in a dwarf galaxy system (Sagittarius: Sgr) based on an analysis of highresolution $H$-band spectra from the Apache Point Observatory Galactic Evolution Experiment (APOGEE) survey. We add [N/Fe], $[\mathrm{Ti} / \mathrm{Fe}]$, and $[\mathrm{Ni} / \mathrm{Fe}]$ abundance ratios to the existing sample of potential members of $\mathrm{M} 54$; this is the first time that $[\mathrm{N} / \mathrm{Fe}]$ abundances are derived for a large number of stars in M 54. Our study reveals the existence of a significant population of nitrogen- (with a large spread, $\gtrsim 1$ dex) and aluminum-enriched stars with moderate Mg depletion in the core of the M 54+Sagittarius system, which shares the light element anomalies characteristic of second-generation globular cluster stars (GCs), thus tracing the typical phenomenon of multiple stellar populations seen in other Galactic GCs at similar metallicity, confirming earlier results based on the Na-O anticorrelation. We further show that most of the stars in M 54 exhibit different chemical - patterns evidently not present in Sgr field stars. Furthermore, we report the serendipitous discovery of a nitrogen-enhanced extra-tidal star with GC second-generation-like chemical patterns for which both chemical and kinematic evidence is commensurate with the hypothesis that the star has been ejected from M 54. Our findings support the existence of chemical anomalies associated with likely tidally shredded GCs in dwarf galaxies in the Local Group and motivate future searches for such bonafide stars along other known Milky Way streams.
\end{abstract}

Key words. stars: abundances - stars: chemically peculiar - globular clusters: individual: M 54 - techniques: spectroscopic

\section{Introduction}

The Sagittarius (Sgr) dwarf spheroidal (dSph) galaxy is one of the closest massive satellites of the Milky Way (MW) (Ibata et al. 1994), and has yielded a wealth of observational evidence of ongoing accretion by the MW in the form of persistent stellar debris and tidal streams discovered by Mateo et al. (1996), and extensively studied with photometric and spectroscopic observations over a huge range of distances $(\sim 10-100 \mathrm{kpc})$ (see, e.g., Ibata et al. 2001; de Boer et al. 2015) using different stellar tracers-including Carbon stars (Totten \& Irwin 1998), the first all-sky map of the tails using 2MASS M-giants (Majew-

\footnotetext{
$\star$ To whom correspondence should be addressed; E-mail jose.fernandez@uda.cl and/or jfernandezt87@gmail.com
}

ski et al. 2003), red clump Stars (Correnti et al. 2010), RR Lyrae stars (Newberg et al. 2003; Ramos et al. 2020), and CN-strong stars (Hanke et al. 2020), among other tracers, usually in small patches along the stream (see, e.g., Li et al. 2019). These studies have been followed-up by numerical studies (see, e.g., Law et al. 2005; Vasiliev et al. 2020), as well as by using precise astrometry from the Gaia second data release (Gaia DR2; Gaia Collaboration et al. 2018a), based on proper motions alone (Antoja et al. 2020). Its proximity provides a unique laboratory to study accretion in detail, through the tidally stripped streams that outflow from the Sgr system (Hasselquist et al. 2017, 2019; Hayes et al. 2020).

As a natural result of such an accretion event, there is a claim in the literature that not only field stars but also GCs have been 

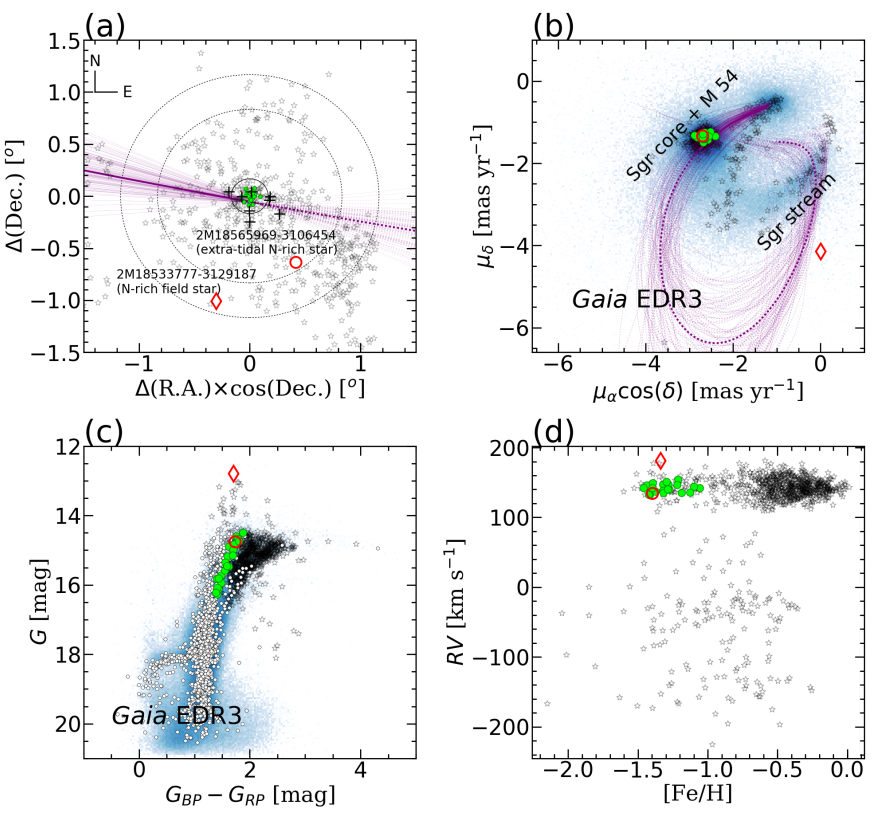

Fig. 1. Panel (a): Spatial positions of the stars in our sample, with the tidal radius $\left(r_{t}=10^{\prime}\right)$ of M 54 over-plotted with a solid line. The open red symbols designate $\mathrm{N}$-rich stars (the diamond symbol refers to a field star, while the open circle highlights the extra-tidal member of M 54). The lime circles designate the M 54 population analyzed in this work, while the black plus symbols designate the stars analyzed by Nataf et al. (2019). The empty grey 'star' symbols designate the potential Sgr population from Hayes et al. (2020). The two concentric circles indicate 5 $r_{t}$ and $7 r_{t}$ for reference. Panel (b): Gaia EDR3 proper motions of stars that have been associated with the Sgr stream: blue symbols for the Antoja et al. (2020) stars and open black 'star' symbols for Hayes et al (2020) stars. The orbital path of Sgr is shown by the dotted (backward) and solid (forward) purple line in panels (a) and (b), with the thick and thin lines showing the central orbit, and one hundred ensemble of orbits that shows the more probable regions of the space, which are crossed more frequently by the simulated orbit, respectively. Panel (c): Color magnitude diagram from Gaia EDR3 photometry of our sample. The symbols are the same as in panels (a) and (b), except the white circles, which denotes the M 54 members from Gaia EDR3, selected on proper motions and within $3^{\prime}$ from the cluster center. Panel (d): Radial velocities versus $[\mathrm{Fe} / \mathrm{H}]$ ratios determined from APOGEE-2/ASPCAP (black symbols) and our $[\mathrm{Fe} / \mathrm{H}]$ ratio determinations from BACCHUS (green and red symbols) in the field around $\mathrm{M} 54$. The $[\mathrm{Fe} / \mathrm{H}]$ APOGEE-2/ASPCAP determinations have been systematically offset by $\sim 0.11$ dex in order to compare with our $[\mathrm{Fe} / \mathrm{H}]$ BACCHUS determinations, as suggested in (Fernández-Trincado et al. 2020c).

accreted (see, e.g., Massari et al. 2019). Some have been speculated to be lost in the disruption process, and may lie immersed in the Sgr stream. Candidates include: M 54, Terzan 7, Arp 2, Terzan 8, Pal 12, Whiting 1, NGC 2419, NGC 6534, and NGC 4147 (e.g., Law \& Majewski 2010; Bellazzini et al. 2020), but a firm connection is still under debate (e.g., Villanova et al. 2016; Tang et al. 2018; Huang \& Koposov 2020; Yuan et al. 2020). In this context, "chemical tagging" (e.g., Freeman \& BlandHawthorn 2002), which is based on the principle that the photospheric chemical compositions of stars reflect the site of their formation, is a promising route for investigation of this question.

While the abundances of light and heavy elements for individual stars in GCs have been widely explored (e.g., Pancino et al. 2017; Mészáros et al. 2020), little is known about these abundances in disrupted GCs likely associated with the closest dwarf galaxies, such as Sgr (Karlsson et al. 2012). Although some evidence for chemical anomalies has been detected towards the inner bulge and halo of the MW (see, e.g., FernándezTrincado et al. 2016; Recio-Blanco et al. 2017; Schiavon et al. 2017; Fernández-Trincado et al. 2017) and Local Group dwarf galaxies (see, e.g., Fernández-Trincado et al. 2020b), suggesting the presence of GCs in the form of disrupted remnants, alternative ways to produce these stars have been recently discussed (Bekki 2019).

This paper is outlined as follows. The high-resolution spectroscopic observations are discussed in Section 2. Section 2.1 describes the sample associated with M 54, including a comparison with data from the literature. Section 3 presents our estimated stellar parameters and derived chemical-abundance determinations. Section 4 discusses the results, and our concluding remarks are presented in Section 5.

\section{Data}

We make use of the internal dataset (which includes all data taken through March 2020) of the second-generation Apache Point Observatory Galactic Evolution Experiment (APOGEE2; Majewski et al. 2017), which includes the first observations from the Irénée du Pont 2.5-m Telescope at Las Campanas Observatory (APO-2S; Bowen \& Vaughan 1973) in the Southern Hemisphere (Chile), and more observations from the Sloan 2.5m Telescope at Apache Point Observatory (Gunn et al. 2006, APO-2N;) in the Northern Hemisphere (New Mexico). The survey operates with two nearly identical spectrographs (Eisenstein et al. 2011; Wilson et al. 2012, 2019), collecting highresolution $(R \sim 22,000)$ spectra in the near-infrared textitHband (1.5145-1.6960 $\mu \mathrm{m}$, vacuum wavelengths). This data set provides stellar parameters, chemical abundances, and radial velocity (RV) information for more than 600,000 sources, which include $\sim 437,000$ targets from the sixteenth data release (DR16; Ahumada et al. 2020) of the fourth generation of the Sloan Digital Sky Survey (SDSS-IV; Blanton et al. 2017). APOGEE-2 target selection is described in full detail in Zasowski et al. (2017) (APOGEE-2), Santana et al. (in prep.) (APO-2S), and Beaton et al. (in prep.) (APO-2N).

APOGEE-2 spectra were reduced (Nidever et al. 2015) and analyzed using the APOGEE Stellar Parameters and Chemical Abundance Pipeline (ASPCAP; García Pérez et al. 2016; Holtzman et al. 2015, 2018; Jönsson et al. 2018, 2020). The model grids for APOGEE-2 internal dataset are based on a complete set of MARCS stellar atmospheres (Gustafsson et al. 2008), which now extend to effective temperatures as low as $3200 \mathrm{~K}$, and spectral synthesis using the Turbospectrum code (Plez 2012). The APOGEE-2 spectra provide access to more than 26 chemical species, which are described in Smith et al. (2013), Shetrone et al. (2015), Hasselquist et al. (2016), Cunha et al. (2017), and Holtzman et al. (2018).

\subsection{54 field}

The APOGEE-2 field toward M 54 was previously examined in Mészáros et al. (2020) based on public DR16 spectra. In that work, 22 stars were identified as potential members linked to M 54 based in the APOGEE-2 radial velocities (Nidever et al. 2015), i.e., stars with RV within $3 \sigma_{R V \text {,cluster }}$, metallicity within \pm 0.5 dex around the cluster average, proper motion from the Gaia Early Data Release 3 (Gaia EDR3; Gaia Collaboration 
et al. 2020) within $2.5 \sigma$ around the cluster average proper motion, and located inside the cluster tidal radius, $r_{t} \lesssim 10$ arcmin, (Harris 1996, 2010 edition) were classified as potential members of M 54. However, only 7 out of 22 stars were spectroscopically examined with the BACCHUS code in Masseron et al. (2016), since only these stars achieved a signal-to-noise $(\mathrm{S} / \mathrm{N}>70)$ sufficient to provide reliable abundance determinations.

The post-APOGEE DR16 dataset provides incremental visits toward M 54, which has allowed to increase the signal-to-noise for 20 out of 22 of the potential cluster members. As a result, nitrogen, titanium, and nickel abundances can be now obtained from the stronger absorption features (as shown for ${ }^{12} \mathrm{C}^{14} \mathrm{~N}$ lines as shown in Figure A.1), and other chemical species can also be studied.

Nataf et al. (2019), using APOGEE-2 DR14 data (Abolfathi et al. 2018) and abundance determinations from the Payne pipeline (Ting et al. 2019), have catalogued eight possible members from M 54. Two of those objects (2M18544275-3029012 and 2M18550740-3026052) were included in our study. The remaining six stars were rejected from our analysis for the following reasons. Six objects in Nataf et al. (2019) were found to have low $\mathrm{S} / \mathrm{N}(<70)$ spectra, resulting in very uncertain $\mathrm{CNO}$ abundance ratios for many chemical species, since the molecular lines $\left({ }^{16} \mathrm{OH},{ }^{12} \mathrm{C}^{16} \mathrm{O}\right.$, and $\left.{ }^{12} \mathrm{C}^{14} \mathrm{~N}\right)$ are very weak. Secondly, 6 out of the 8 objects in Nataf et al. (2019) exhibit $[\mathrm{Fe} / \mathrm{H}]>-1.1$, and were recently classified as Sgr stars (see, e.g., Hayes et al. 2020), which make them unlikely members of M 54.

In this study, we make use of the more recent spectra to examine the chemical composition of added stars to the abundance average of M 54. As in Mészáros et al. (2020), we also limit our discussion only to stars with $\mathrm{S} / \mathrm{N}>70$.

\subsection{Extra-tidal stars}

We also report on the serendipitous discovery of two nitrogenenhanced (N-rich) metal-poor stars beyond the tidal radius of M 54, as shown in pane (a) of Figure 1. APOGEE-2 stars in the stream+core Sagittarius (Sgr) system (see, e.g., Hasselquist et al. 2017, 2019; Hayes et al. 2020) are highlighted as black open 'star' symbols in pane (a) of Figure 1, while potential star members (blue symbols) of the stream+core Sgr system from Antoja et al. (2020) are also displayed in panel (a) of Figure 1. It is important to note that the $[\mathrm{Fe} / \mathrm{H}]$ abundance of APOGEE-2 Sgr stars are provided by the ASPCAP pipeline (see Hasselquist et al. 2017, 2019; Hayes et al. 2020). In order to compare with our $[\mathrm{Fe} / \mathrm{H}]$ determinations, an offset of $\sim 0.11$ dex was applied to ASPCAP metallicities in panel (d) of Figure 1, as suggested in Fernández-Trincado et al. (2020c).

Panels (a) to (d) of Figure 1 reveal that one (2M18565969-3106454) of the newly discovered N-rich stars meets the minimum criterion to be considered a potential extra-tidal star which has likely escaped the cluster potential, while the second N-rich star (2M18533777-3129187) has physical properties that are clearly offset from the M 54 population. In particular, this star is brighter than the typical population of M 54 (see panel (c) in Figure 1), and both proper motions and RV differ from the nominal proper motion and RV of the cluster as shown in panels (b) and (d) of Figures 1. It is likely that 2M18533777-3129187 is a foreground field star (hereafter N-rich field star).

\section{Stellar parameters and chemical-abundance determinations}

The chemical analysis is very similar to that carried out by Fernández-Trincado et al. (2019a,b,c,d, 2020a,b,c,e, 2021b). The stellar parameters $\left(T_{\text {eff }}, \log g\right.$, and first guess on metallicity) for the 20 cluster members with $\mathrm{S} / \mathrm{N}>70$ were extracted from Mészáros et al. (2020), while we adopt the atmospheric parameters from the uncalibrated post-APOGEE DR16 values for the two stars beyond the cluster tidal radius. The elemental abundances and final errors in $[\mathrm{Fe} / \mathrm{H}]$ and $[\mathrm{X} / \mathrm{Fe}]$, astrometric and kinematic properties of our sample are listed in Tables A.1, A.2, and A.3, respectively.

A consistent chemical-abundance analysis was then carried out with the BACCHUS code (Masseron et al. 2016), from which we obtained the metallicities from Fe I lines, and abundances for twelve other chemical species belonging to the light- $(\mathrm{C}, \mathrm{N})$, $\alpha-(\mathrm{O}, \mathrm{Mg}, \mathrm{Si}, \mathrm{Ca}$, and Ti), Fe-peak (Ni), odd-Z (Al, K) and $s$ process $(\mathrm{Ce}, \mathrm{Nd})$ elements.

\section{Results and discussion}

Panel (a) of Figure 2 summarizes the chemical enrichment seen in M 54 stars analyzed in this work, and compares to the Mészáros et al. (2020) determinations. The chemical composition of the two newly identified N-rich stars beyond the cluster tidal radius is also shown in the same figure. Overall, the chemical abundance of M 54 based on the added cluster stars is within the typical errors, and does not affect the science results presented in Mészáros et al. (2020), while the two external N-rich stars share chemical patterns similar to the M 54 population.

For $\mathrm{M} 54$, we find a mean metallicity $\langle[\mathrm{Fe} / \mathrm{H}]\rangle=-1.30 \pm$ 0.12 , which agrees well with Mészáros et al. $(2020)^{1}$. The spread in $[\mathrm{Fe} / \mathrm{H}]$ increased from 0.04 to $0.12 \mathrm{dex}$, but it is still smaller than that reported in Carretta et al. (2010). Even if the measured scatter is larger than that reported by Mészáros et al. (2020), it does not seem to indicate the presence of a significant spread in $[\mathrm{Fe} / \mathrm{H}]$, and is similar to that observed in Galactic globular clusters (GCs) at similar metallicity, such as M 10 (see, e.g., Mészáros et al. 2020). Nickel (an element that belongs to the Fegroup), exhibits a flat distribution as a function of $[\mathrm{Fe} / \mathrm{H}]$, similar to that observed in Carretta et al. (2010), and at odds with that observed in Sgr stars.

Regarding the other chemical species, we find excellent agreement with the values provided by Mészáros et al. (2020), as can be seen in panel (a) of Figure 2, with the main difference that the added stars introduce a larger star-to-star scatter than previously measured. M 54 exhibits a modest enhancement in $\alpha$-elements, with mean values for $[\mathrm{O} / \mathrm{Fe}],[\mathrm{Mg} / \mathrm{Fe}]$, $[\mathrm{Si} / \mathrm{Fe}],[\mathrm{Ca} / \mathrm{Fe}]$, and $[\mathrm{Ti} / \mathrm{Fe}]$ which is similar to what is seen in halo GCs: $\langle[\mathrm{O} / \mathrm{Fe}]\rangle=+0.64 \pm 0.36$ (14 stars); $\langle[\mathrm{Mg} / \mathrm{Fe}]\rangle=$ $+0.18 \pm 0.11$ (18 stars); $\langle[\mathrm{Si} / \mathrm{Fe}]\rangle=+0.26 \pm 0.10$ (20 stars); $\langle[\mathrm{Ca} / \mathrm{Fe}]\rangle=+0.25 \pm 0.07$ (16 stars); and the new measured $\langle[\mathrm{Ti} / \mathrm{Fe}]\rangle=+0.21 \pm 0.21$ (16 stars), indicating a fast enrichment provided by supernovae ( $\mathrm{SNe}$ ) II events. Mean values are in good agreement with Mészáros et al. (2020), with the exception of oxygen, which displays the larger star-to-star spread expected in likely second-generation stars.

We also find that the $[\mathrm{O} / \mathrm{Fe}],[\mathrm{Mg} / \mathrm{Fe}]$, and $[\mathrm{Si} / \mathrm{Fe}]$ ratios are almost flat as a function of the metallicity, while $[\mathrm{Ca} / \mathrm{Fe}]$ and

\footnotetext{
1 Note that here, and for the abundances described below, the number following the average abundance represents the one-sigma dispersion, not the error in the mean.
} 

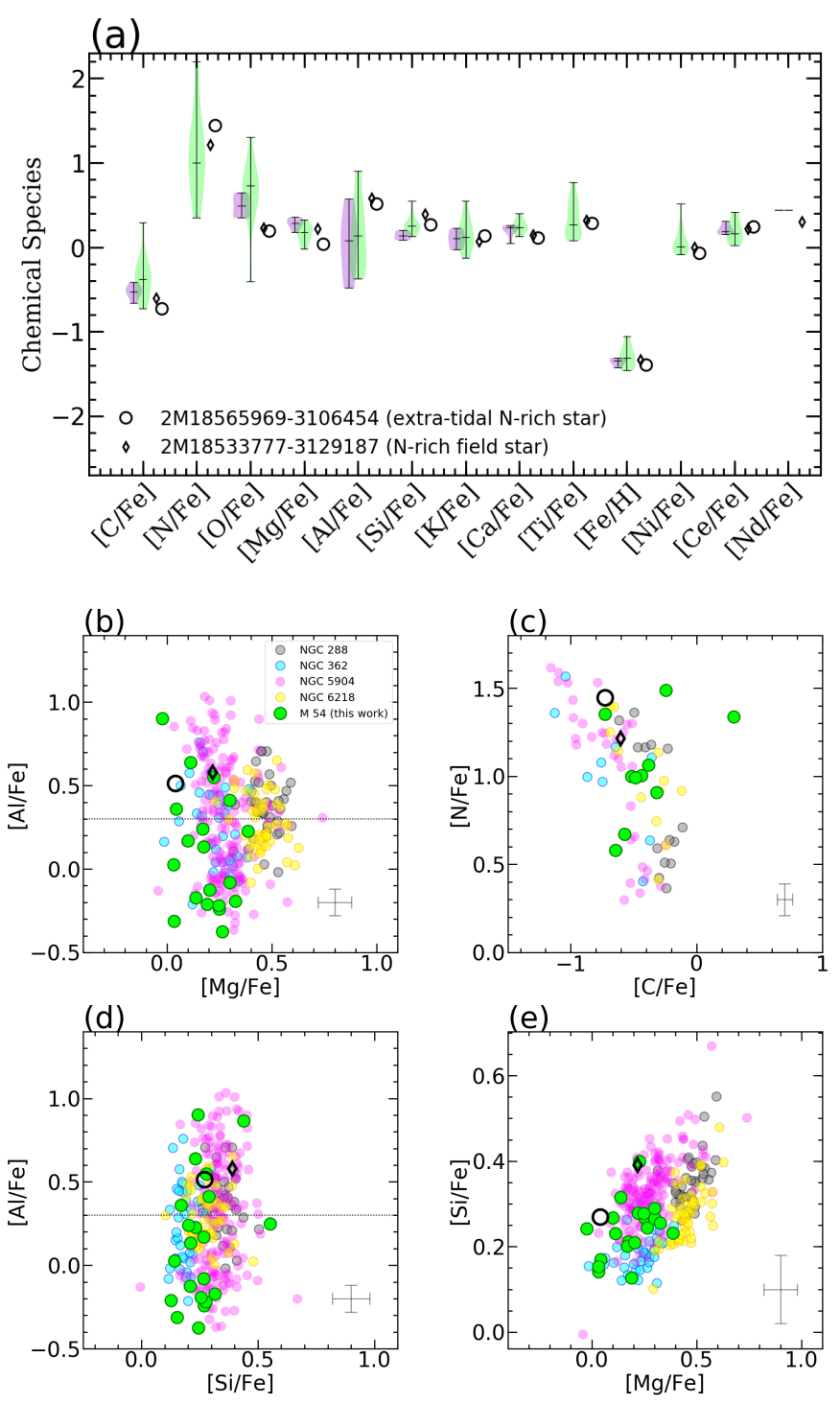

Fig. 2. BACCHUS elemental abundances. Panel (a): The observed $[\mathrm{X} / \mathrm{H}]$ and $[\mathrm{Fe} / \mathrm{H}]$ abundance-density estimation (violin representation) of M 54 stars, and the observed abundance ratios of newly identified N-rich stars. The extra-tidal star from M 54 and a field star is highlighted with a black open circle and diamond, respectively. Each violin indicates with horizontal lines the median and limits of the distribution. The lime and dark violet violin representation refer to the abundance ratios of 20 stars (this work) and 7 stars from Mészáros et al. (2020), respectively. Panels (b)-(e): Distributions of light- $(\mathrm{C}, \mathrm{N}), \alpha-(\mathrm{Mg}, \mathrm{Si})$ and odd-Z (Al) elements in different abundance planes. In each panel, the planes $[\mathrm{Al} / \mathrm{Fe}]-[\mathrm{Mg} / \mathrm{Fe}],[\mathrm{N} / \mathrm{Fe}]-[\mathrm{C} / \mathrm{Fe}],[\mathrm{Al} / \mathrm{Fe}]-[\mathrm{Si} / \mathrm{Fe}],[\mathrm{Si} / \mathrm{Fe}]-$ $[\mathrm{Mg} / \mathrm{Fe}]$ are shown, respectively, for GCs from Mészáros et al. (2020). The black dotted line at $[\mathrm{Al} / \mathrm{Fe}]=+0.3$ indicates the separation of $\mathrm{FG}$ and SG stars as proposed in Mészáros et al. (2020). The distribution of M 54 stars (lime squares) analyzed in this work are overlaid. The black open circle and diamond refer to the extra-tidal and field N-rich star, respectively. The plotted error bars show the typical abundance uncertainties.

$[\mathrm{Ti} / \mathrm{Fe}]$ ratios slightly increases as $[\mathrm{Fe} / \mathrm{H}]$ increases, similar to the behaviour found by Carretta et al. (2010). On the contrary, the $\alpha$-element trend observed in Sgr stars (see, e.g., Carretta et al. 2010; McWilliam et al. 2013; Hasselquist et al. 2017, 2019) differ from those seen in the population of M 54. Overall, the $\alpha$-elements in the cluster are higher than seen in Sgr stars. In conclusion, the measured $\alpha$-enrichment in this work support the previous hypothesis suggesting that the $\alpha$-element in M 54 stars formed before the typical $e$-folding time for $\mathrm{SN}$ Ia contributing their ejecta to the gas pool (e.g., Carretta et al. 2010).

We also found that some stars in M 54 appear to be quite $\mathrm{Mg}$ poor, with strong enrichment in aluminum and nitrogen, providing further evidence for the presence of second-generation stars in M 54, and the signature of very high temperatures achieved during H-burning (e.g., Carretta et al. 2010; Mészáros et al. 2020). The odd- $Z$ elements ( $\mathrm{Al}$ and $\mathrm{K}$ ) in $\mathrm{M} 54$ exhibit an average $\langle[\mathrm{Al} / \mathrm{Fe}]\rangle=+0.14 \pm 0.37$ (19 stars) and $\langle[\mathrm{K} / \mathrm{Fe}]\rangle=$ $+0.15 \pm 0.18$ (17 stars), with a clear anti-correlation in Al-Mg, as can be seen in in panel (b) of Figure 2, with moderate $\mathrm{Mg}$ depletions related to the enrichment in $\mathrm{Al}$ abundances, as the result of the conversion of $\mathrm{Mg}$ into $\mathrm{Al}$ during the $\mathrm{Mg}$ - $\mathrm{Al}$ cycle (e.g., Carretta et al. 2010; Denissenkov et al. 2015; Renzini et al. 2015; Pancino et al. 2017). This pattern is evidently not present in the Sgr stars, where, on the contrary, ASPCAP Mg and Al abundances are positively correlated with each other (see, e.g., Hasselquist et al. 2017, 2019; Hayes et al. 2020).

We derived average abundances for $\mathrm{C}$ and $\mathrm{N}$ in $\mathrm{M} 54$, of $\langle[\mathrm{C} / \mathrm{Fe}]\rangle=-0.36 \pm 0.25(13$ stars $)$ and $\langle[\mathrm{N} / \mathrm{Fe}]\rangle=+1.12 \pm$ 0.48 (17 stars). Most of the stars in M 54 are $\mathrm{C}$ deficient $([\mathrm{C} / \mathrm{Fe}] \lesssim+0.3)$ and $\mathrm{N}$ enhanced $([\mathrm{N} / \mathrm{Fe}]>+0.5)$, but they do not exhibit the typical N-C anti-correlation (see panel (c) of Figure 2) seen in other GCs at similar metallicity (e.g. Mészáros et al. 2020), most probably due the lack of stars with low nitrogen abundances. On the contrary, an apparent continuous distribution of $\mathrm{N}$ abundances is present in $\mathrm{M} 54$. This result indicates the prevalence of the multiple-population phenomenon in M 54 as previously suggested in the literature (Carretta et al. 2010; Milone et al. 2017; Sills et al. 2019; Mészáros et al. 2020).

Additionally, we do not find any evidence for the presence of the $\mathrm{K}-\mathrm{Mg}$ anti-correlation in M 54, as have been suggested to be present in a few Galactic GCs at similar metallicity (Mészáros et al. 2020). Furthermore, a Si-Al correlation is slightly evident in M 54, as shown in panel (d) of Figure 2, and has a stubby Mg$\mathrm{Si}$ distribution (see panel (e) of Figure 2), which is an indication of ${ }^{28} \mathrm{Si}$ production from the result of a secondary leakage in the main Mg-Al cycle, which is instead absent in the Sgr stars.

For the elements produced by neutron $(n)$-capture processes (Ce II and Nd II), we find, on average, $\langle[\mathrm{Ce} / \mathrm{Fe}]\rangle=+0.18 \pm 0.13$ (10 stars) and $[\mathrm{Nd} / \mathrm{Fe}]=+0.44$ ( 1 star). Overall, M 54 exhibits a modest enrichment in $s$-process elements, with a few stars as enhanced as +0.4 , similar to that observed in Galactic GC stars at similar metallicity (see, e.g., Mészáros et al. 2020), suggesting that it is possible that the $s$-process enrichment has been produced by a different source than the progenitor of the $\mathrm{Mg}-\mathrm{Al}$ anti-correlations, possibly by low-mass asymptotic giant branch stars. Lastly, we find that $[\mathrm{Ce} / \mathrm{Fe}]$ ratios in M 54 are almost flat as a function of metrallicity. Unfortunately, $\mathrm{Nd}$ II is measured in only one star, which has been found to exhibit the modest enhancement, consistent with a moderate enrichment of $s$-process elements.

Furthermore, we report the serendipitous discovery of two $\mathrm{N}$-enhanced stars identified within $\sim 7 r_{t}$ from M 54, as shown in panel (a) of Figure 1. Panel (a) of Figure 2 show the collection of $[\mathrm{X} / \mathrm{Fe}]$ and $[\mathrm{Fe} / \mathrm{H}]$ abundance ratios for the two newly identified $\mathrm{N}$-rich stars beyond the tidal radius of M 54. Both stars exhibit very similar chemical-abundance patterns as those seen in the population of M 54. A plausible explanation is that both stars were previous members of M 54, from which they have been ejected. However, this possibility seems unlikely for one of these 
extra-tidal stars (2M18533777-3129187), which was ruled out as a possible member of $\mathrm{M} 54$.

As can be appreciated from inspection of panels (a) to (d) of Figure 1, the current position of 2M18533777-3129187 does not resemble the kinematic and astrometric properties (e.g., Antoja et al. 2020; Hayes et al. 2020) of Sgr+M 54 stars, nor the orbital path of $\mathrm{Sgr}^{2}$. It is also the most luminous star in our sample, making it a likely foreground star. The possibility that this star was disrupted from M 54 and deposited in the inner Galaxy seems unlikely, as the perigalacticon of M 54 is located well beyond the solar radius (see, e.g., Baumgardt et al. 2019). We conclude that 2M18533777-3129187 is a N-enhanced field star born in a different progenitor than M 54, but with a similar chemical-enrichment history to this cluster.

Aside from 2M18533777-3129187, there is another $\mathrm{N}$ enhanced field star (2M18565969-3106454) located $\sim 5 \times r_{t}$ from the cluster center, which exhibits a stellar atmosphere strongly enriched in nitrogen $([\mathrm{N} / \mathrm{Fe}]>+1.4)$, as extreme as M 54 stars, accompanied by a very low carbon abundance $([\mathrm{C} / \mathrm{Fe}]<-0.7)$, and with discernible contributions from the $s$ process elements $(\mathrm{Ce} \mathrm{II})$. Since the $[\mathrm{Al} / \mathrm{Fe}]$ ratio is $>+0.5$, which is a 'typical' value for stars in GCs, and unlikely in dwarf galaxy populations, we conclude that 2M18565969-3106454 shares the same nucleosynthetic pathways of second-generation stars in M 54.

2M18565969-3106454 is a potential extra-tidal star with kinematics and astrometric properties similar to that of M 54 stars, and exhibits unique chemical patterns comparable to that of genuine second-generation GC stars, which makes it very different from Sgr stars. On the other hand, N-rich stars are commonly observed to be more centrally concentrated in GCs (e.g. Dalessandro et al. 2019) and as a consequence they have smaller probabilities to be tidally stripped. Thus, it is likely that the extra-tidal star could well be just a stripped M 54 star as many others in its surroundings. Our finding demonstrate that $\mathrm{N}$-rich stars are a promising route for identifying the unambiguous chemical signatures of stars formed in GC-like environment which may lie immersed in the M 54+Sgr core and/or Sgr stream, as well as confirm or discard the possible association of GCs to the Sgr stream (Bellazzini et al. 2020).

Following the same methodology as described in FernándezTrincado et al. (2021a), we compute the predicted number $\left(N_{N-r i c h}\right)$ of N-rich field stars observed in APOGEE-2 toward M 54/Sgr using the smooth halo density relations presented in Horta et al. (2021), and by adopting the same Monte Carlo implementation of the Von Neumann Rejection Technique (see e.g., Press et al. 2002) as in Eq. 7 in (Fernández-Trincado et al. 2015). We find the expected number of observed N-rich halo stars beyond $d_{\odot} \gtrsim 15 \mathrm{kpc}$ over the sky area of 1.5 degree radius centred in M 54, and with both astrometric and kinematic properties as M 54 to be $N_{N-\text { rich }}<0.1$ (from 1000 Monte Carlo realisations). This yield a very low probability that the new identified extra-tidal N-rich star associated with M 54 is due to random fluctuations in the field. Furthermore, we also use the Besancon galactic model (Robin et al. 2003) and the GravPot16 model (Fernández-Trincado et al. 2020d) to explore the expectations for

\footnotetext{
2 The Sgr orbit was computed with the GravPot16 model, https: //gravpot.utinam.cnrs. fr, by adopting the same model configurations as described in Fernández-Trincado et al. (2020c). For the Sgr centre, we adopt the heliocentric distance $d_{\odot}=26.5 \mathrm{kpc}$ and heliocentric radial velocity $R V=142 \mathrm{~km} \mathrm{~s}^{-1}$ from Vasiliev \& Belokurov (2020), and proper motions from Gaia Collaboration et al. (2018b): $\mu_{\alpha} \cos \delta=-2.692$ mas $\mathrm{yr}^{-1}$ and $\mu_{\delta}=-1.359$ mas $\mathrm{yr}^{-1}$, with uncertainties assumed of the order of $10 \%$ in $d_{\odot}, R V$, and proper motions.
}

a "default" Milky Way along the RVs to the Sgr+M5̃4 surrounding field beyond $d_{\odot} \gtrsim 15 \mathrm{kpc}$. The "all" sample is dominated by halo kinematics with a negligible contribution from the thin and thick disk beyond $R V \gtrsim 120 \mathrm{~km} \mathrm{~s}^{-1}$. Thus, our Milky Way simulated sample act to guide us in $R V$ space, confirming that the kinematics of the newly identified extra-tidal N-rich star differs from the disk population, with practically low contribution form the expected halo. ,

\section{Concluding remarks}

We present a spectroscopic analysis for 20 out 22 red giant stars that are members of M 54 from the internal APOGEE DR16 dataset. This study doubles the sample of stars with spectroscopic measurements for this cluster, and the new post-APOGEE DR16 spectra achieve high signal-to-noise ( $\mathrm{S} / \mathrm{N}>70)$, allowing the addition of new chemical species not examined in previous studies (e.g., Mészáros et al. 2020) in the $H$-band-APOGEE-2 footprint.

Overall, the chemical species re-examined in M 54 were found to be consistent with previous studies (Mészáros et al. 2020), although most of them exhibit a large star-to-star scatter. We find that 15 out of the 20 stars investigated show a high $[\mathrm{N} / \mathrm{Fe}]$ abundance ratio $([\mathrm{N} / \mathrm{Fe}] \gtrsim+0.5)$, confirming the prevalence of the MPs phenomenon in M 54. Both [Ni/Fe] and [Ti/Fe], not previously examined in Mészáros et al. (2020), were found to be in good agreement with measurements in the literature. In particular, we confirm the $[\mathrm{Ti} / \mathrm{Fe}]$ ratio slightly increases as $[\mathrm{Fe} / \mathrm{H}]$ increases, as has been reported in Carretta et al. (2010). We also find a large spread in $[\mathrm{Al} / \mathrm{Fe}]$, and the presence of a genuine second-generation star in M 54, which exhibits $\mathrm{Mg}$ deficiency $([\mathrm{Mg} / \mathrm{Fe}]<0)$ accompanied with large enhancements in nitrogen and aluminum. In general, all chemical species examined in the M 54 members present distinguishable chemical behaviour compared with Sgr stars, suggesting a different chemical-evolution history that resembles other Galactic halo GCs at similar metallicity.

Furthermore, we report on the serendipitous discovery of a potential extra-tidal star toward the surrounding regions of the M 54+Sgr core, which exhibits a strong enrichment in nitrogen comparable to that seen in M 54 stars. As far as we know this is the first study reporting on the unambiguous chemical signatures of stars formed in GC-like environment into a nearby satellite dwarf galaxy around the Milky Way. Finding out how many of such chemical unusual stars likely originated in GCs are present in dwarf galaxy systems, help to understand the link between GCs and their stellar streams (see e.g., Bellazzini et al. 2020).

Acknowledgements. The author is grateful for the enlightening feedback from the anonymous referee. J.G.F-T is supported by FONDECYT No. 3180210. T.C.B. acknowledges partial support for this work from grant PHY 14-30152: Physics Frontier Center / JINA Center for the Evolution of the Elements (JINA-CEE), awarded by the US National Science Foundation. D.M. is supported by the BASAL Center for Astrophysics and Associated Technologies (CATA) through grant AFB 170002, and by project FONDECYT Regular No. 1170121. S.V. gratefully acknowledges the support provided by Fondecyt regular No. 1170518. D.G. gratefully acknowledges support from the Chilean Centro de Excelencia en Astrofísica y Tecnologías Afines (CATA) BASAL grant AFB-170002. D.G. also acknowledges financial support from the Dirección de Investigación y Desarrollo de la Universidad de La Serena through the Programa de Incentivo a la Investigación de Académicos (PIA-DIDULS). A.R.-L. acknowledges financial support provided in Chile by Agencia Nacional de Investigación y Desarrollo (ANID) through the FONDECYT project 1170476. B.B. acknowledge partial financial support from the Brazilian agencies CAPES-Financial code 001, CNPq, and FAPESP.

This work has made use of data from the European Space Agency (ESA) mission Gaia (http://www.cosmos.esa.int/gaia), processed by the Gaia Data Processing and Analysis Consortium (DPAC, 
http://www . cosmos.esa.int/web/gaia/dpac/consortium). Funding for the DPAC has been provided by national institutions, in particular the institutions participating in the Gaia Multilateral Agreement.

Funding for the Sloan Digital Sky Survey IV has been provided by the Alfred P. Sloan Foundation, the U.S. Department of Energy Office of Science, and the Participating Institutions. SDSS- IV acknowledges support and resources from the Center for High-Performance Computing at the University of Utah. The SDSS web site is www.sdss.org. SDSS-IV is managed by the Astrophysical Research Consortium for the Participating Institutions of the SDSS Collaboration including the Brazilian Participation Group, the Carnegie Institution for Science, Carnegie Mellon University, the Chilean Participation Group, the French Participation Group, Harvard-Smithsonian Center for Astrophysics, Instituto de Astrofissica de Canarias, The Johns Hopkins University, Kavli Institute for the Physics and Mathematics of the Universe (IPMU) / University of Tokyo, Lawrence Berkeley National Laboratory, Leibniz Institut für Astrophysik Potsdam (AIP), Max-Planck-Institut für Astronomie (MPIA Heidelberg), Max-Planck-Institut für Astrophysik (MPA Garching), Max-Planck-Institut für Extraterrestrische Physik (MPE), National Astronomical Observatory of China, New Mexico State University, New York University, University of Notre Dame, Observatório Nacional / MCTI, The Ohio State University, Pennsylvania State University, Shanghai Astronomical Observatory, United Kingdom Participation Group, Universidad Nacional Autónoma de México, University of Arizona, University of Colorado Boulder, University of Oxford, University of Portsmouth, University of Utah, University of Virginia, University of Washington, University of Wisconsin, Vanderbilt University, and Yale University.

\section{References}

Abolfathi, B., Aguado, D. S., Aguilar, G., et al. 2018, ApJS, 235, 42 Ahumada, R., Allende Prieto, C., Almeida, A., et al. 2020, ApJS, 249, 3

Antoja, T., Ramos, P., Mateu, C., et al. 2020, A\&A, 635, L3

Baumgardt, H., Hilker, M., Sollima, A., \& Bellini, A. 2019, MNRAS, 482, 5138 Bekki, K. 2019, MNRAS, 490, 4007

Bellazzini, M., Ibata, R., Malhan, K., et al. 2020, A\&A, 636, A107

Blanton, M. R., Bershady, M. A., Abolfathi, B., et al. 2017, AJ, 154, 28

Bowen, I. S. \& Vaughan, A. H., J. 1973, Appl. Opt., 12, 1430

Carretta, E., Bragaglia, A., Gratton, R. G., et al. 2010, A\&A, 520, A95

Correnti, M., Bellazzini, M., Ibata, R. A., Ferraro, F. R., \& Varghese, A. 2010, ApJ, 721, 329

Cunha, K., Smith, V. V., Hasselquist, S., et al. 2017, ApJ, 844, 145

Dalessandro, E., Cadelano, M., Vesperini, E., et al. 2019, ApJ, 884, L24

de Boer, T. J. L., Belokurov, V., \& Koposov, S. 2015, MNRAS, 451, 3489

Denissenkov, P. A., VandenBerg, D. A., Hartwick, F. D. A., et al. 2015, MNRAS, 448,3314

Eisenstein, D. J., Weinberg, D. H., Agol, E., et al. 2011, AJ, 142, 72

Fernández-Trincado, J. G., Beers, T. C., \& Minniti, D. 2020a, A\&A, 644, A83

Fernández-Trincado, J. G., Beers, T. C., Minniti, D., et al. 2020b, ApJ, 903, L17

Fernández-Trincado, J. G., Beers, T. C., Minniti, D., et al. 2021a, arXiv e-prints, arXiv:2102.01706

Fernández-Trincado, J. G., Beers, T. C., Minniti, D., et al. 2020c, A\&A, 643, L4

Fernández-Trincado, J. G., Beers, T. C., Placco, V. M., et al. 2019a, ApJ, 886, L8

Fernández-Trincado, J. G., Beers, T. C., Tang, B., et al. 2019b, MNRAS, 488, 2864

Fernández-Trincado, J. G., Chaves-Velasquez, L., Pérez-Villegas, A., et al. 2020d, MNRAS, 495, 4113

Fernández-Trincado, J. G., Mennickent, R., Cabezas, M., et al. 2019c, A\&A, 631, A97

Fernández-Trincado, J. G., Minniti, D., Beers, T. C., et al. 2020e, A\&A, 643, A 145

Fernández-Trincado, J. G., Minniti, D., Souza, S. O., et al. 2021b, arXiv e-prints, arXiv:2102.01088

Fernández-Trincado, J. G., Robin, A. C., Moreno, E., et al. 2016, ApJ, 833, 132

Fernández-Trincado, J. G., Vivas, A. K., Mateu, C. E., et al. 2015, A\&A, 574 A15

Fernández-Trincado, J. G., Zamora, O., García-Hernández, D. A., et al. 2017, ApJ, 846, L2

Fernández-Trincado, J. G., Zamora, O., Souto, D., et al. 2019d, A\&A, 627, A178

Freeman, K. \& Bland-Hawthorn, J. 2002, ARA\&A, 40, 487

Gaia Collaboration, Brown, A. G. A., Vallenari, A., et al. 2018a, A\&A, 616, A1

Gaia Collaboration, Brown, A. G. A., Vallenari, A., et al. 2020, arXiv e-prints, arXiv:2012.01533

Gaia Collaboration, Helmi, A., van Leeuwen, F., et al. 2018b, A\&A, 616, A12

García Pérez, A. E., Allende Prieto, C., Holtzman, J. A., et al. 2016, AJ, 151, 144

Gunn, J. E., Siegmund, W. A., Mannery, E. J., et al. 2006, AJ, 131, 2332
Gustafsson, B., Edvardsson, B., Eriksson, K., et al. 2008, A\&A, 486, 951

Hanke, M., Koch, A., Prudil, Z., Grebel, E. K., \& Bastian, U. 2020, A\&A, 637, A98

Harris, W. E. 1996, AJ, 112, 1487

Hasselquist, S., Carlin, J. L., Holtzman, J. A., et al. 2019, ApJ, 872, 58

Hasselquist, S., Shetrone, M., Cunha, K., et al. 2016, ApJ, 833, 81

Hasselquist, S., Shetrone, M., Smith, V., et al. 2017, ApJ, 845, 162

Hayes, C. R., Majewski, S. R., Hasselquist, S., et al. 2020, ApJ, 889, 63

Holtzman, J. A., Hasselquist, S., Shetrone, M., et al. 2018, AJ, 156, 125

Holtzman, J. A., Shetrone, M., Johnson, J. A., et al. 2015, AJ, 150, 148

Horta, D., Mackereth, J. T., Schiavon, R. P., et al. 2021, MNRAS, 500, 5462

Huang, K.-W. \& Koposov, S. E. 2020, MNRAS[arXiv: 2005. 14014]

Ibata, R., Irwin, M., Lewis, G. F., \& Stolte, A. 2001, ApJ, 547, L133

Ibata, R. A., Gilmore, G., \& Irwin, M. J. 1994, Nature, 370, 194

Jönsson, H., Allende Prieto, C., Holtzman, J. A., et al. 2018, AJ, 156, 126

Jönsson, H., Holtzman, J. A., Allende Prieto, C., et al. 2020, AJ, 160, 120

Karlsson, T., Bland-Hawthorn, J., Freeman, K. C., \& Silk, J. 2012, ApJ, 759, 111

Law, D. R., Johnston, K. V., \& Majewski, S. R. 2005, ApJ, 619, 807

Law, D. R. \& Majewski, S. R. 2010, ApJ, 718, 1128

Li, J., FELLOW, L., Liu, C., et al. 2019, ApJ, 874, 138

Majewski, S. R., Schiavon, R. P., Frinchaboy, P. M., et al. 2017, AJ, 154, 94

Majewski, S. R., Skrutskie, M. F., Weinberg, M. D., \& Ostheimer, J. C. 2003 , ApJ, 599, 1082

Massari, D., Koppelman, H. H., \& Helmi, A. 2019, A\&A, 630, L4

Masseron, T., Merle, T., \& Hawkins, K. 2016, BACCHUS: Brussels Automatic Code for Characterizing High accUracy Spectra

Mateo, M., Mirabal, N., Udalski, A., et al. 1996, ApJ, 458, L13

McWilliam, A., Wallerstein, G., \& Mottini, M. 2013, ApJ, 778, 149

Mészáros, S., Masseron, T., García-Hernández, D. A., et al. 2020, MNRAS, 492, 1641

Milone, A. P., Piotto, G., Renzini, A., et al. 2017, MNRAS, 464, 3636

Nataf, D. M., Wyse, R. F. G., Schiavon, R. P., et al. 2019, AJ, 158, 14

Newberg, H. J., Yanny, B., Grebel, E. K., et al. 2003, ApJ, 596, L191

Nidever, D. L., Holtzman, J. A., Allende Prieto, C., et al. 2015, AJ, 150, 173

Pancino, E., Romano, D., Tang, B., et al. 2017, A\&A, 601, A112

Plez, B. 2012, Turbospectrum: Code for spectral synthesis

Press, W. H., Teukolsky, S. A., Vetterling, W. T., \& Flannery, B. P. 2002, Numerical recipes in $\mathrm{C}++:$ the art of scientific computing

Ramos, P., Mateu, C., Antoja, T., et al. 2020, A\&A, 638, A104

Recio-Blanco, A., Rojas-Arriagada, A., de Laverny, P., et al. 2017, A\&A, 602, L14

Renzini, A., D'Antona, F., Cassisi, S., et al. 2015, MNRAS, 454, 4197

Robin, A. C., Reylé, C., Derrière, S., \& Picaud, S. 2003, A\&A, 409, 523

Schiavon, R. P., Zamora, O., Carrera, R., et al. 2017, MNRAS, 465, 501

Shetrone, M., Bizyaev, D., Lawler, J. E., et al. 2015, ApJS, 221, 24

Sills, A., Dalessandro, E., Cadelano, M., Alfaro-Cuello, M., \& Kruijssen, J. M. D. 2019, MNRAS, 490, L67

Smith, V. V., Cunha, K., Shetrone, M. D., et al. 2013, ApJ, 765, 16

Tang, B., Fernández-Trincado, J. G., Geisler, D., et al. 2018, ApJ, 855, 38

Ting, Y.-S., Conroy, C., Rix, H.-W., \& Cargile, P. 2019, ApJ, 879, 69

Totten, E. J. \& Irwin, M. J. 1998, MNRAS, 294, 1

Vasiliev, E. \& Belokurov, V. 2020, MNRAS, 497, 4162

Vasiliev, E., Belokurov, V., \& Erkal, D. 2020, MNRAS[arXiv: 2009. 10726]

Villanova, S., Monaco, L., Moni Bidin, C., \& Assmann, P. 2016, MNRAS, 460, 2351

Wilson, J. C., Hearty, F., Skrutskie, M. F., et al. 2012, in Society of Photo-Optical Instrumentation Engineers (SPIE) Conference Series, Vol. 8446, Groundbased and Airborne Instrumentation for Astronomy IV, ed. I. S. McLean, S. K. Ramsay, \& H. Takami, $84460 \mathrm{H}$

Wilson, J. C., Hearty, F. R., Skrutskie, M. F., et al. 2019, PASP, 131, 055001

Yuan, Z., Chang, J., Beers, T. C., \& Huang, Y. 2020, ApJ, 898, L37

Zasowski, G., Cohen, R. E., Chojnowski, S. D., et al. 2017, AJ, 154, 198

Article number, page 6 of 10 


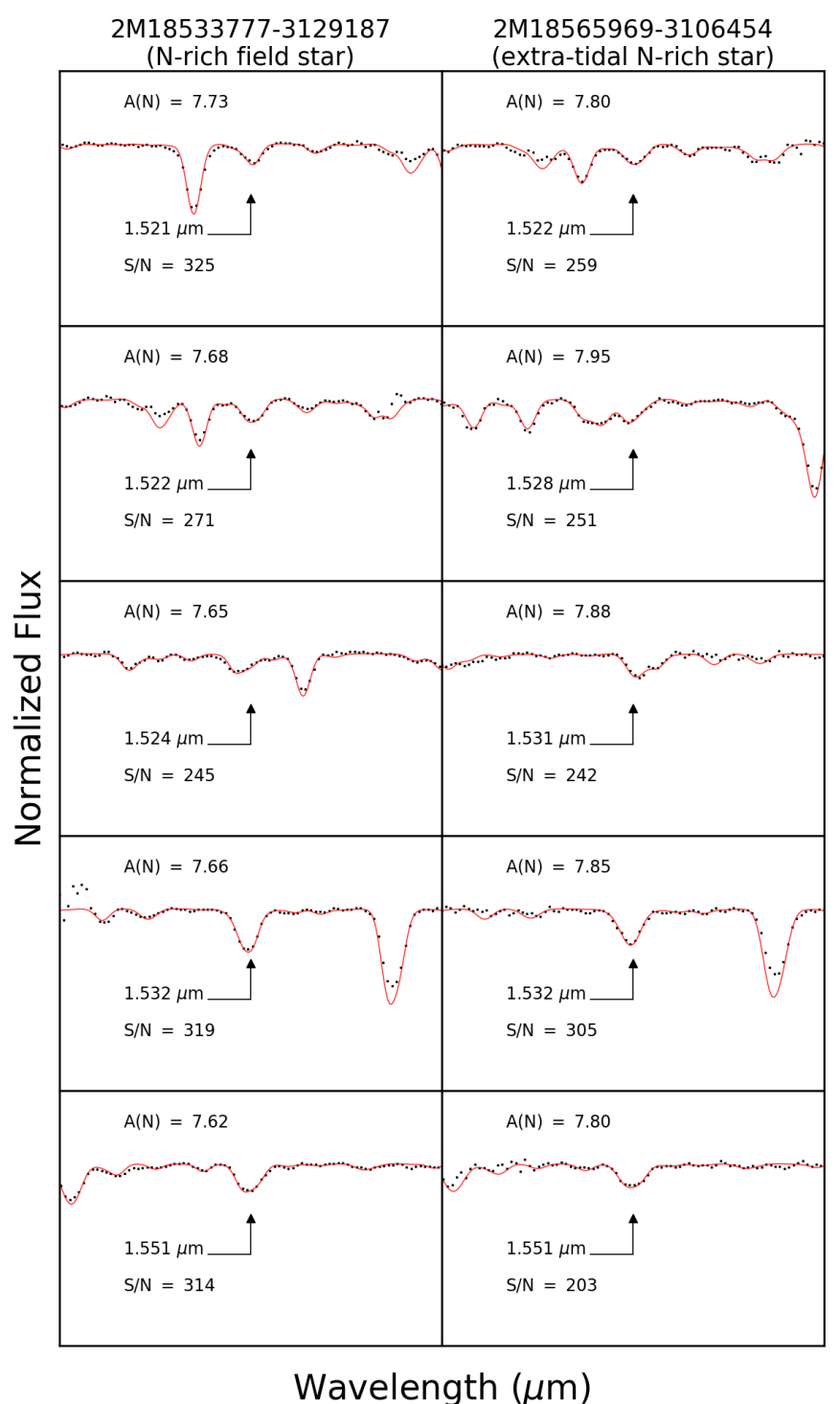

Fig. A.1. Detection of ${ }^{12} \mathbf{C}^{14} \mathbf{N}$ lines. Spectral synthesis for the determination of nitrogen abundances for two $\mathrm{N}$-rich stars beyond the tidal radius of M 54. Each panel shows the best-fit syntheses (red lines) from BACCHUS compared to the observed spectra (black squares) of selected ${ }^{12} \mathrm{C}^{14} \mathrm{~N}$ lines (grey arrows)

\section{Appendix A: Spectrum of N-rich stars}

Figure A.1 shows an example of the local thermodynamic equilibrium (LTE) -BACCHUS spectral synthesis of selected ${ }^{12} \mathrm{C}^{14} \mathrm{~N}$ lines for the two newly identified N-rich stars beyond the tidal radius of M 54. The black squares represent the observed spectrum, and the solid red line is the best abundance fit.

Table A.1 and A.2 list the atmospheric parameters, abundance ratios and the final errors in $[\mathrm{Fe} / \mathrm{H}]$ and $[\mathrm{X} / \mathrm{Fe}]$, while Table A.3 list of the main physical properties of our sample. 
A\&A proofs: manuscript no. ms

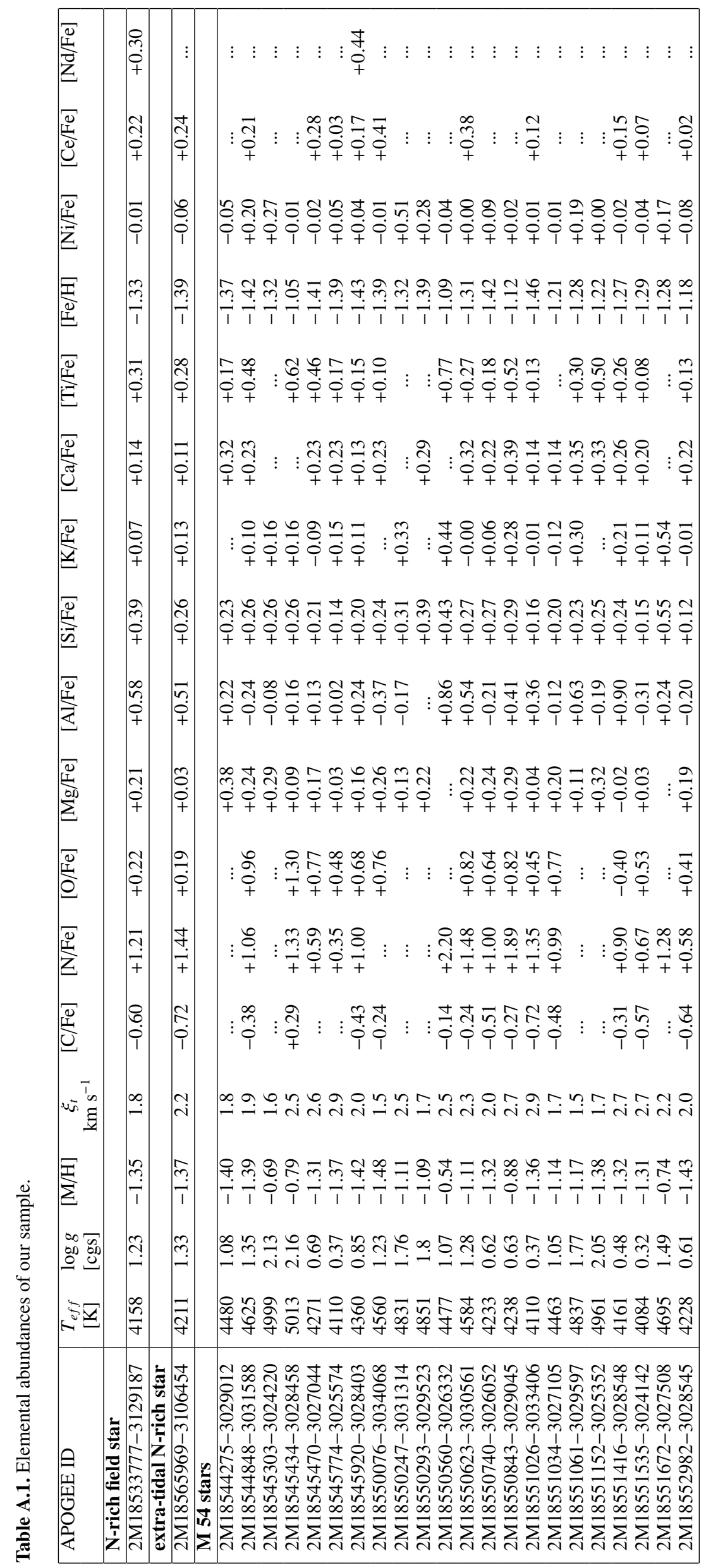




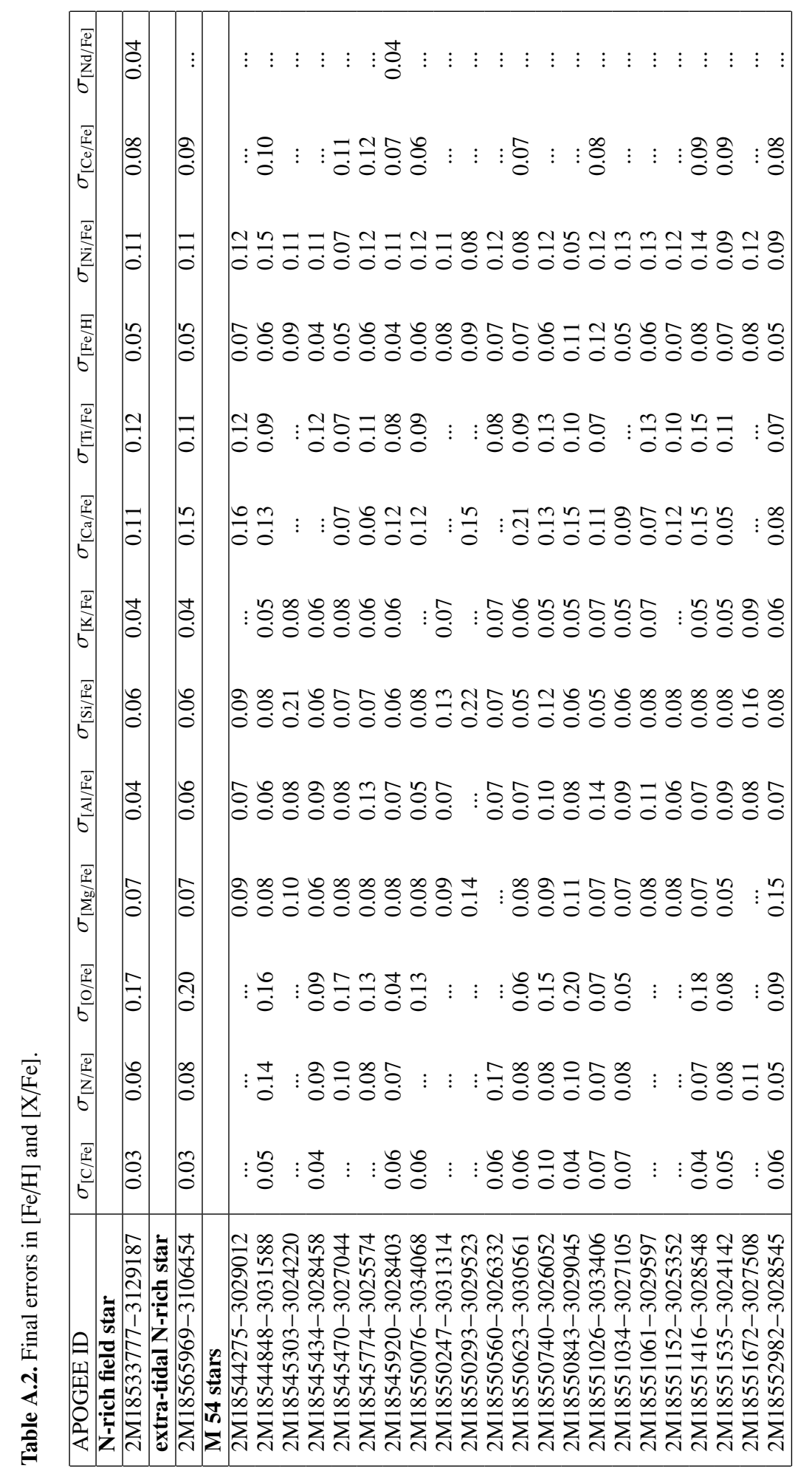


Table A.3. Astrometric and kinematic properties of our sample. The last two columns indicate the typical S/N of the spectra and number of APOGEE visits.

\begin{tabular}{|c|c|c|c|c|c|c|c|c|c|c|}
\hline APOGEE ID & RUWE & $\begin{array}{c}\mu_{\alpha} \cos (\delta) \pm \Delta \\
\operatorname{mas} \mathrm{yr}^{-1}\end{array}$ & $\begin{array}{c}\mu_{\delta} \pm \Delta \\
\operatorname{mas}_{\mathrm{yr}^{-1}}\end{array}$ & $\begin{array}{c}G \\
{[\mathrm{mag}]}\end{array}$ & $\begin{array}{c}G_{B P} \\
{[\mathrm{mag}]}\end{array}$ & $\begin{array}{c}G_{R P} \\
{[\mathrm{mag}]}\end{array}$ & $\begin{array}{c}R V \\
\mathrm{~km} \mathrm{~s}^{-1}\end{array}$ & $\begin{array}{c}R V \text {-scatter } \\
\mathrm{km} \mathrm{s}^{-1}\end{array}$ & $\begin{array}{c}\text { S/N } \\
\text { pixel }^{-1}\end{array}$ & \# Visits \\
\hline N-rich field star & & & & & & & & \multicolumn{3}{|c|}{ pixel $^{-1}$} \\
\hline 2M18533777-3129187 & 0.9 & $0.001 \pm 0.016$ & $-4.150 \pm 0.013$ & 12.79 & 13.62 & 11.91 & 181.02 & 0.26 & 402 & 6 \\
\hline \multicolumn{11}{|l|}{ extra-tidal N-rich star } \\
\hline 2M18565969-3106454 & 0.9 & $-2.701 \pm 0.027$ & $-1.336 \pm 0.023$ & 14.75 & 15.59 & 13.86 & 134.75 & 0.55 & 225 & 12 \\
\hline \multicolumn{11}{|l|}{ M 54 stars } \\
\hline 2M18544275-3029012 & 1.0 & $-2.667 \pm 0.040$ & $-1.357 \pm 0.033$ & 15.55 & 16.27 & 14.71 & 137.07 & 0.33 & 51 & 4 \\
\hline 2M18544848-3031588 & 1.1 & $-2.686 \pm 0.037$ & $-1.356 \pm 0.030$ & 15.17 & 15.91 & 14.32 & 141.74 & 0.34 & 172 & 12 \\
\hline $2 \mathrm{M} 18545303-3024220$ & 0.8 & $-2.584 \pm 0.052$ & $-1.430 \pm 0.045$ & 16.27 & 16.91 & 15.49 & 147.14 & 0.91 & 53 & 10 \\
\hline 2M18545434-3028458 & 1.1 & $-2.694 \pm 0.052$ & $-1.440 \pm 0.045$ & 15.72 & 16.33 & 14.85 & 142.11 & 0.42 & 109 & 12 \\
\hline 2M18545470-3027044 & 1.4 & $-2.412 \pm 0.035$ & $-1.345 \pm 0.030$ & 14.60 & 15.44 & 13.68 & 131.88 & 1.16 & 266 & 12 \\
\hline 2M18545774-3025574 & 1.1 & $-2.717 \pm 0.029$ & $-1.321 \pm 0.025$ & 14.68 & 15.54 & 13.75 & 148.86 & 1.76 & 262 & 12 \\
\hline 2M18545920-3028403 & 1.1 & $-2.800 \pm 0.040$ & $-1.374 \pm 0.035$ & 14.88 & 15.54 & 13.92 & 145.79 & 0.71 & 217 & 12 \\
\hline 2M18550076-3034068 & 0.9 & $-2.685 \pm 0.036$ & $-1.494 \pm 0.030$ & 15.35 & 16.07 & 14.52 & 149.07 & 0.38 & 118 & 6 \\
\hline 2M18550247-3031314 & 1.0 & $-2.625 \pm 0.049$ & $-1.310 \pm 0.040$ & 16.10 & 16.76 & 15.33 & 138.48 & 0.77 & 82 & 12 \\
\hline 2M18550293-3029523 & 1.0 & $-2.526 \pm 0.045$ & $-1.230 \pm 0.037$ & 15.82 & 16.30 & 14.89 & 141.96 & 0.49 & 115 & 12 \\
\hline 2M18550560-3026332 & 0.9 & $-2.617 \pm 0.045$ & $-1.345 \pm 0.038$ & 15.93 & 16.60 & 15.12 & 143.86 & 0.29 & 93 & 10 \\
\hline 2M18550623-3030561 & 1.0 & $-2.832 \pm 0.038$ & $-1.366 \pm 0.032$ & 15.57 & 16.29 & 14.74 & 135.17 & 0.31 & 136 & 12 \\
\hline 2M18550740-3026052 & 1.0 & $-2.658 \pm 0.028$ & $-1.243 \pm 0.023$ & 14.82 & 15.65 & 13.94 & 132.96 & 0.95 & 93 & 4 \\
\hline 2M18550843-3029045 & 1.0 & $-2.557 \pm 0.038$ & $-1.340 \pm 0.031$ & 15.46 & 16.16 & 14.54 & 135.85 & 0.35 & 158 & 12 \\
\hline 2M18551026-3033406 & 1.1 & $-2.644 \pm 0.027$ & $-1.407 \pm 0.021$ & 14.49 & 15.41 & 13.54 & 142.01 & 0.74 & 309 & 12 \\
\hline 2M18551034-3027105 & 1.0 & $-2.682 \pm 0.037$ & $-1.379 \pm 0.030$ & 15.44 & 16.19 & 14.60 & 154.11 & 0.31 & 106 & 6 \\
\hline 2M18551061-3029597 & 1.1 & $-2.888 \pm 0.054$ & $-1.325 \pm 0.044$ & 16.07 & 16.65 & 15.24 & 147.11 & 0.94 & 78 & 11 \\
\hline 2M18551152-3025352 & 1.0 & $-2.665 \pm 0.053$ & $-1.407 \pm 0.044$ & 16.22 & 16.74 & 15.35 & 146.85 & 0.62 & 73 & 10 \\
\hline 2M18551416-3028548 & 1.1 & $-2.675 \pm 0.031$ & $-1.342 \pm 0.026$ & 14.98 & 15.80 & 14.10 & 139.67 & 0.38 & 208 & 12 \\
\hline 2M18551535-3024142 & 1.0 & $-2.683 \pm 0.029$ & $-1.403 \pm 0.023$ & 14.74 & 15.62 & 13.82 & 151.11 & 1.01 & 230 & 12 \\
\hline 2M18551672-3027508 & 0.9 & $-2.692 \pm 0.043$ & $-1.407 \pm 0.035$ & 15.80 & 16.44 & 15.00 & 140.11 & 0.94 & 76 & 11 \\
\hline 2M18552982-3028545 & 0.9 & $-2.689 \pm 0.033$ & $-1.292 \pm 0.028$ & 15.15 & 15.96 & 14.27 & 135.09 & 0.38 & 192 & 12 \\
\hline
\end{tabular}

